\title{
USO DE HABITAT DE CUATRO CARNIVOROS TERRESTRES EN EL SUR DE CHILE
}

\section{HABITAT USE OF FOUR TERRESTRIAL CARNIVORES IN SOUTHERN CHILE}

\author{
Alfredo Zúñiga, Andrés Muñoz-Pedreros \& Andrés Fierro \\ Laboratorio de Ecología Aplicada y Biodiversidad, Escuela de Ciencias Ambientales, Facultad de Recursos Naturales, \\ Universidad Católica de Temuco, Casilla 15-D, Temuco, Chile. \\ E-mail: zundusicyon@gmail.com

\section{RESUMEN}

El proceso de transformación del paisaje ha sido más intenso y vasto en la depresión intermedia del centro y sur de Chile que en las zonas cordilleranas aledañas. En dicha depresión el bosque nativo original prácticamente ha desaparecido y en el sur de Chile ha permanecido fragmentado e inserto en una matriz de agroecosistemas. Esto puede influir en la distribución de los mamíferos carnívoros, según sean especialistas o generalistas de hábitat, así como del tamaño de su ámbito de hogar. En este contexto el objetivo de este estudio fue evaluar, mediante el estudio de la distribución de fecas, la selección de hábitat del ensamble de carnívoros en un ambiente fragmentado en el sur de Chile. Documentamos el uso selectivo de Puma concolor, Galictis cuja y Lycalopex griseus de las plantaciones forestales exóticas con matorrales. Leopardus guigna, a pesar de no mostrar una selección estadísticamente significativa por el bosque nativo, presenta mayor número de registros en dicho hábitat. La selección de hábitat de los depredadores estudiados muestra un grado variable de uso de los ambientes intervenidos y fragmentados. En las plantaciones forestales circundantes existe un sotobosque de vegetación nativa que se adecua a los requerimientos de la mayoría de los carnívoros. Se discuten la superposición espacial y la adecuación a nuevos ambientes.

Palabras Clave: Carnívoros, fragmentación, selección de hábitat, sobreposición espacial.

\section{ABSTRACT}

The process of landscape transformation has been more intense and extended in the central depression of central and southern Chile than in the nearby mountain ranges. The original forest cover has almost disappeared; only small fragments persist inserted into a matrix of agroecosystems. This situation may influence the distribution of carnivorous mammals, depending on their degree of habitat specialization and home range size. The goal of this study was to evaluate, through the study of feces dsitribution, the habitat selection of the carnivore assemblage in a fragmented environment in southern Chile. We document the selective use of exotic forest plantations of Pinus radiata with a scrub understory by Puma concolor, Galictis cuja, and Lycalopex griseus. Leopardus guigna, despite not showing a statistically significant selection of the native forest, presents a greater number of records in this type of habitat. Habitat selection by the predators studied shows a variable degree of use of altered and fragmented environments. Surrounding forest plantations present an understory of native vegetation, which fits the requirement of most of the carnivores. The levels of spatial overlapping and the adequacy to new environments are discussed.

KEYwords: Carnivores, fragmentation, habitat selection, spatial overlapping, 
Uso de hábitat por carnívoros en el sur de Chile: ZúÑIGA, A. ET AL.

\section{INTRODUCCION}

La fragmentación del bosque nativo conlleva a la división de los hábitats originales en fragmentos de tamaño menor y rodeados por matrices estructural y funcionalmente distintas, lo que puede modificar la biodiversidad original (Skole \& Tucker 1993; Crooks 2002; Gehring \& Swinhart 2003; Lindenmayer \& Fischer 2006). En Chile, en los últimos 25 años, este proceso ha sido intenso y vasto en los valles centrales del centro y sur del país y en menor medida en las zonas cordilleranas aledañas (San Martín \& Donoso 1996; Echeverría et al. 2006). En las zonas más intervenidas, el bosque nativo original prácticamente ha desaparecido y ha sido reemplazado por cultivos agrícolas y plantaciones forestales con especies exóticas, incluyendo Pinus radiata (D.Don) y Eucalyptus globulus (Labill) (véase Echeverria et al. 2006, 2008).

La forma e intensidad con que la fragmentación del bosque nativo afecta a las poblaciones de mamíferos va a depender, entre otros factores, de los requerimientos de hábitat de cada una (Caughley 1994; Virgós 2002; Virgós et al. 2002), de sus adecuaciones conductuales (e.g., caza, territorialidad, gregariedad) (Kleiman \& Eisenberg 1973) y de la manera en que cada especie se vincula con los tipos de cobertura vegetacional existente (Murray et al. 1995). La selección de hábitat en carnívoros no sólo está influenciada por la disponibilidad de presas, sino que también por la oferta de refugios. Es relevante, entonces, poder establecer cómo estos animales se adecúan a los cambios ocurridos a nivel de paisaje y así poder predecir su permanencia a largo plazo en estos ambientes perturbados (Caughley 1994; Carrol et al 2001).

En la provincia de Cautín en la Región de la Araucanía de Chile existen remanentes de vegetación nativa rodeados de una matriz de agroecosistemas donde predominan las áreas de pastoreo y campos de cultivo. El predio Rucamanque (438 hectáreas) comprende un fragmento de bosque nativo que ha sido reconocido como de importancia para la conservación de la biodiversidad regional (Muñoz et al. 1996). En este sitio se han registrado cuatro carnívoros terrestres: el puma Puma concolor (Linnaeus 1771), la guiña Leopardus guigna (Molina 1782), el zorro chilla Lycalopex griseus (Gray 1837) y el quique Galictis cuja (Molina 1782). Se desconoce el uso del hábitat que estas especies hacen en Rucamanque. En otras zonas se han descrito diferentes requerimientos de hábitat para especies de carnívoros nativos, así Acosta \& Simonetti (2004) han documentado que $L$. guigna prefiere fragmentos de bosque nativo sobre plantaciones exóticas. Los zorros, Lycalopex spp., prefieren hábitats abiertos cómo praderas (e.g., Johnson \& Franklin 1994) y plantaciones exóticas (Acosta \& Simonetti 2004). El puma utiliza zonas ecotonales con praderas, matorrales y bosque nativo (Muñoz-Pedreros et al. 1990). Por último, G. cuja no ha sido suficientemente estudiado en Chile (aunque véase Delibes et al. 2003). El objetivo de este estudio es determinar la selección de hábitat de las especies de carnívoros en un ambiente fragmentado de la depresión intermedia en el sur de Chile.

\section{MATERIALES Y METODOS}

\section{ARea De estudio}

El área de estudio consideró un total de 568,9 ha, abarcando un predio con vegetación nativa (Rucamanque) y otro con plantaciones exóticas de coníferas. El predio Rucamanque ( $38^{\circ} 39^{\prime} \mathrm{S}-72^{\circ} 36^{\prime}$ W), de 438 ha, se encuentra al noroeste de la ciudad de Temuco, Región de la Araucanía, Chile, sobre la vertiente sur del cordón montañoso Nielol-Huimpil. Rucamanque posee una topografía de relieve montañoso e irregular. Su cubierta vegetal está dominada por: (a) bosque húmedo siempre-verde, que incluye asociaciones vegetacionales de olivillo (e.g., Lapagerio-Aextoxiconetum punctatii) y roblelaurel-lingue (Nothofago-Perseetum), con un 25\% de especies alóctonas y con diez grupos sintáxicos (Magofke 1985; Ramírez 1982; Ramírez et al. 1989a, 1989b), y (b) matorral y pradera de origen antrópico. Este predio está rodeado por plantaciones forestales de pino insigne Pinus radiata (D. Don) (Fig. 1). El fragmento de bosque nativo más cercano y de similar tamaño a Rucamanque se encuentra unos $90 \mathrm{~km}$ al norte.

\section{Metodología}

Se representaron los hábitats del área de estudio mediante tratamiento en SIG de imágenes digitales empleando el software ArcView 3.2 y su extensión Spatial Analyst 2.0, obteniéndose así una carta de hábitats y la superficie (en ha) de cada uno los mismos. Los tipos de cubierta vegetal identificados fueron: bosque adulto denso, bosque adulto 


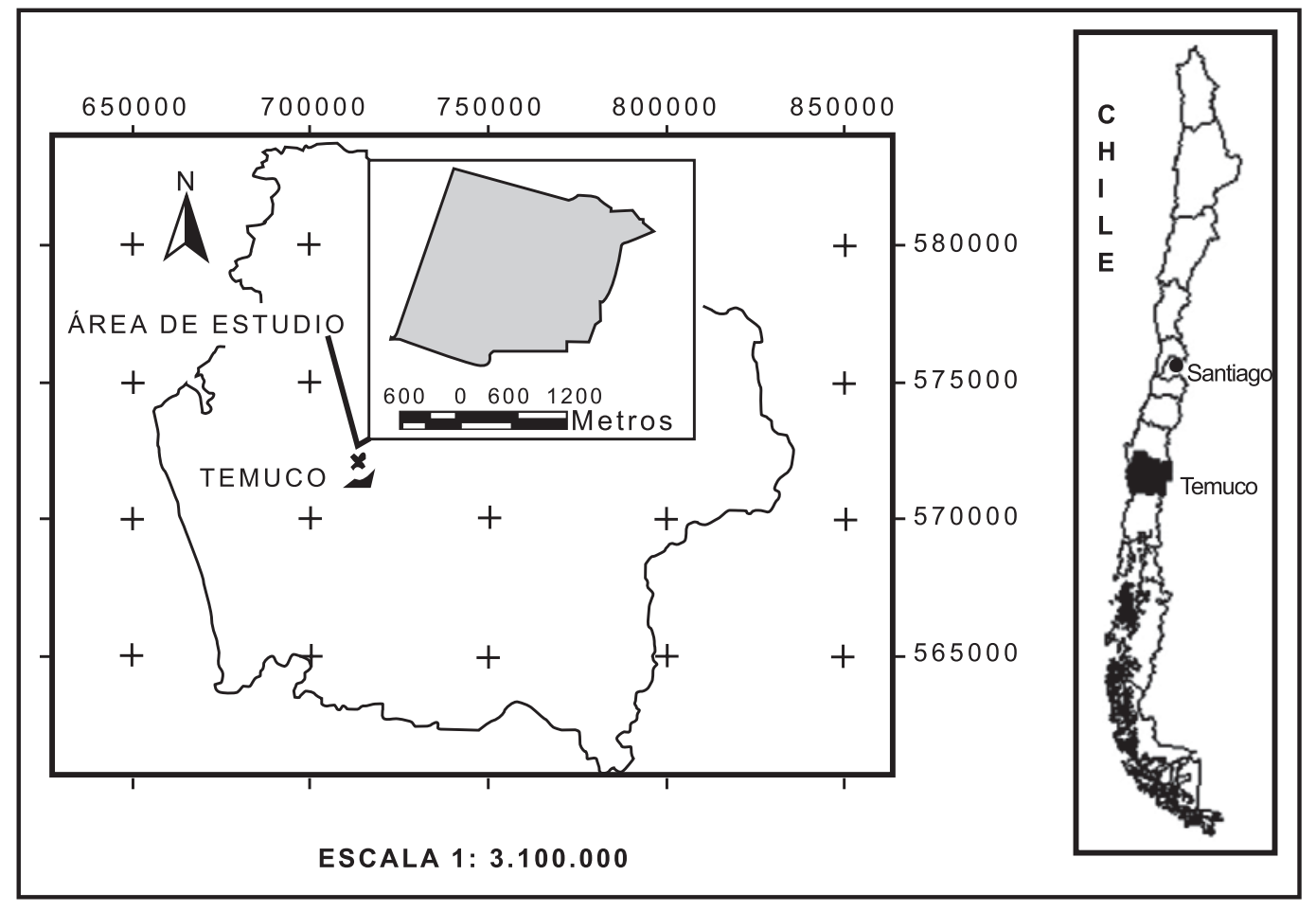

Figura 1. Area de estudio en el predio Rucamanque, Región de la Araucanía, Chile.

FiguRE 1. Study area at predio Rucamanque, Region de la Araucania, Chile.

ralo, renoval de Nothofagus obliqua (Mirb.), matorral, pradera con estrato arbustivo, praderas y plantaciones de $P$. radiata con sotobosque. Los mismos se reunieron en tres ambientes (Fig. 2): (a) bosque nativo (denso, ralo y renovales; 48,50\%), (b) pradera/matorral $(24,17 \%)$ y (c) plantaciones de $P$. radiata $(27,32 \%)$.

La presencia de los cuatro carnívoros estudiados fue cuantificada mediante el registro de fecas (técnica considerada satisfactoria por Sadlier et al. 2004). Las fecas se recogieron a lo largo de 12 transectos de una longitud promedio de $1 \mathrm{~km}$ (total $14 \mathrm{~km}$ ) y cinco metros de ancho. Estos transectos cubrieron la totalidad del área de estudio y en forma equitativa los tres ambientes considerados. Los transectos se recorrieron quincenalmente durante dieciocho meses (noviembre de 2004 a abril de 2006). Sólo se consideraron las fecas íntegras, las que se identificaron según su morfología (Chame 2003); el registro de cada especie identificada por sus fecas se corroboró con registros visuales. También se describieron los ambientes en que se recolectaron las muestras, según su estructura vegetacional dominante (bosque nativo, pradera y plantaciones exóticas de pino). La taxonomía de los mamíferos registrados sigue a Yáñez \& MuñozPedreros (2009).

Para evaluar la selección de hábitat comparamos la frecuencia de fecas observadas de cada especie con la frecuencia esperada, según la proporción de área en cada ambiente, utilizando una prueba de bondad de ajuste de Chi-cuadrado ( $\chi^{2}$ ) (Sokal $\&$ Rohlf 1995), que ha sido utilizada para evaluar selección de hábitat de carnívoros en pequeñas escalas (Sánchez-Lalinde \& Pérez-Torres 2008). Suponemos que si las especies usan los ambientes al azar, la distribución de las fecas sería proporcional a la disponibilidad de hábitat; por el contrario, si hay concentración de las fecas en un(os) ambiente(s) implicaría una selección hacia dichos hábitat(s). La 




FIgURA 2. Cubierta vegetal del predio Rucamanque, Región de la Araucanía, Chile.

FiguRE 2. Plant cover of predio Rucamanque, Region de la Araucania, Chile.

frecuencia esperada se obtuvo relacionando el total de fecas con la proporción de área de cada ambiente. Cuando $\chi^{2}$ fue significativo se determinaron las categorías de hábitat por medio de los intervalos simultáneos de confianza de Bonferroni (Byers et al. 1984) empleando el programa HABUSE 4.0, de tal forma que, en la eventualidad que estos hábitats sean seleccionados positivamente, los intervalos definen el rango de frecuencias de los hábitats que se ocupan. Este procedimiento para determinar uso de hábitat mediante frecuencias de fecas fue validado por Guerrero et al. (2006), quienes además, compararon estas distribuciones de fecas con huellas, empleando estaciones de atracción olfativa, no registrando diferencias significativas entre ambos métodos (véase Acosta-Jamett \& Simonetti 2004). El nivel de significancia de las correlaciones pareadas se obtuvo a través de la corrección de Bonferroni (Holm 1979), con el propósito de compensar el grupo de datos utilizado en varias comparaciones, reduciendo la tasa de error de tipo I.

Respecto a la sobreposición espacial, establecimos un análisis de similitud de hábitat entre las especies de carnívoros, considerando las frecuencias relativas en que sus fecas se presentaron en cada hábitat. Empleamos el índice de distancia ecológica de Bray-Curtis (Odum 1950). Mediante el programa Biodiversity Pro (Mc Aleece 1997). La desviación estándar de este índice fue obtenida mediante el método Jackknife (Jaksic \& Medel 1987). Posteriormente examinamos las correlaciones entre el número de fecas de cada especie en los transectos, empleando el coeficiente de correlación de Pearson (r) (transformada en log; Zar 1984) y siguiendo a Neale \& Sacks (2001), por lo que asumimos que la abundancia relativa de las fecas se corresponde con el uso relativo del espacio, a la vez que la evasión entre los depredadores quedaría insinuada por una correlación negativa entre la frecuencia de sus fecas. 


\section{RESULTADOS}

SELECCIÓn DE HÁBITAT

Fecas de P. concolor, G. cuja y L. griseus fueron encontradas en mayor proporción en plantaciones de $P$. radiata, ambientes que se han descrito como fuente importante de presas (e.g., roedores, lagomorfos y en algunos casos marsupiales) para estos carnívoros. En cambio, fecas de L. guigna fueron mayormente encontradas en bosque nativo, no obstante esta preferencia no fue diferente de lo esperado por azar. En relación a la frecuencia de registros de fecas por hábitat (Fig. 3) y al análisis de Chi-cuadrado para cada especie $\left(\chi^{2}=13,81\right.$; grados de libertad $=2, p=$ $0,05)$ se sugiere que los carnívoros usan los hábitat seleccionados en forma diferente a lo esperado por azar, con excepción de L. guigna (Tabla I). Las plantaciones de $P$. radiata fueron seleccionadas por $P$. concolor, L. griseus y G. cuja. Es destacable para el caso de L. griseus la detección de agregados fecales (letrinas) en el bosque nativo. Asimismo, el bosque nativo fue el hábitat más utilizado por L. guigna (60\% de las frecuencias), a pesar de que no fue seleccionado positivamente de acuerdo a los intervalos de confianza de Bonferroni. La pradera/ matorral fue el hábitat menos utilizado por las cuatro especies ( $<15 \%$ de los registros).

Respecto a las similitudes espaciales, L. guigna es la especie que presenta menor similitud en relación a los otros carnívoros (55\%) (Fig. 4). Por otro lado, L. griseus y P. concolor presentan la mayor similitud en los ambientes que ocupan $(73,8 \%)$ y G. cuja ocupa un valor intermedio a estos valores $(64,4 \%)$. La desviación estándar resultante de estas comparaciones es de 0,117 .

\section{SOBREPOSICIÓN ESPACIAL}

En la Figura 5 se muestra que la correlación de distribución de fecas entre P. concolor y L. griseus tendría una tendencia positiva significativa con un valor de alfa de $0,05(\mathrm{r}=0,730)$, por lo que es improbable su segregación mutua. De la misma manera, podría establecerse esta no segregación entre $P$. concolor y G. cuja $(\mathrm{p}=0,541)$. El resto de las correlaciones entre especies no son significativas. No obstante, al incorporar la corrección de Bonferroni a la significancia $(0,05 / 6=0,083)$ ninguna de las correlaciones resulta significativa.

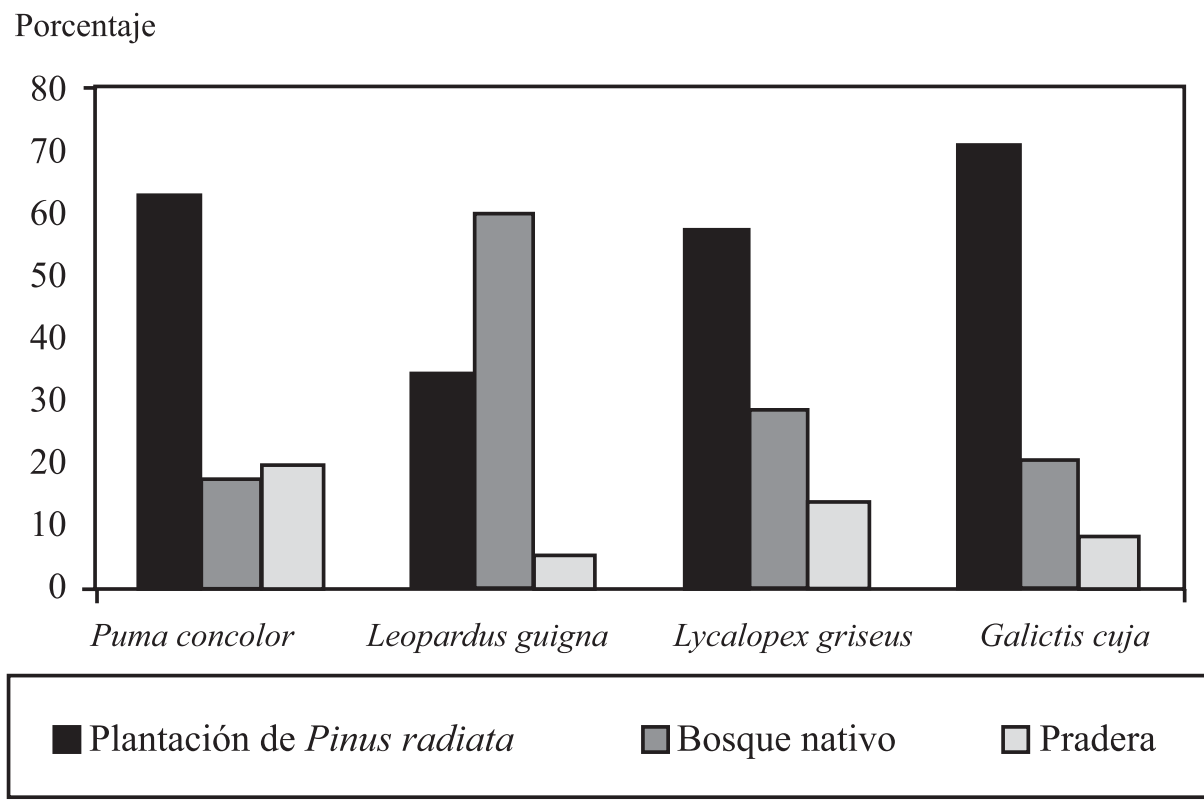

Figura 3. Frecuencias de fecas de cuatro depredadores en diferentes hábitats del predio Rucamanque, Región de la Araucanía. 2005-2006.

FIGURE 3. Frequencies of feces of four predators in different habitats in predio Rucamanque, Region de la Araucania. 2005-2006. 
Uso de hábitat por carnívoros en el sur de Chile: ZúÑIGA, A. ET AL.

TABLA I. Fecas observadas y esperadas de cuatro depredadores en diferentes hábitats del predio Rucamanque, Región de la Araucanía, Chile.

TABLE I. Observed and expected feces of four predators in different habitats at predio Rucamanque, Región de la Araucanía, Chile.

\begin{tabular}{|c|c|c|c|c|c|c|c|c|}
\hline \multirow[b]{2}{*}{ Especie } & \multicolumn{3}{|c|}{ Hábitat } & \multirow[b]{2}{*}{$\begin{array}{c}\text { Estadístico } \\
\chi^{2}\end{array}$} & \multirow[b]{2}{*}{$\begin{array}{l}\text { Total } \\
\text { Fecas }\end{array}$} & \multicolumn{3}{|c|}{$\begin{array}{l}\text { Intervalo de } \\
\text { Bonferroni } \\
p=0.05\end{array}$} \\
\hline & $\begin{array}{l}\text { Plantación } \\
\text { de } P \text {. radiata } \\
\text { (obs/esp) }\end{array}$ & $\begin{array}{l}\text { Bosque } \\
\text { nativo } \\
\text { (obs/esp) }\end{array}$ & $\begin{array}{l}\text { Pradera } \\
\text { (obs/esp) }\end{array}$ & & & $\begin{array}{l}\text { Plantación } \\
\text { de } \\
\text { P. radiata }\end{array}$ & Bosque & Pradera \\
\hline $\begin{array}{l}\text { Puma } \\
\text { concolor }\end{array}$ & $(28 / 12,29)$ & $(8 / 21,82)$ & $(9 / 10,87)$ & 29,15 & 45 & $\begin{array}{c}0,144-0,401 \\
(=)\end{array}$ & $\begin{array}{c}0,341-0,628 \\
(+)\end{array}$ & $\begin{array}{c}0,119-0,364 \\
(=)\end{array}$ \\
\hline $\begin{array}{l}\text { Leopardus } \\
\text { guigna }\end{array}$ & $(12 / 9,56)$ & $(21 / 16,97)$ & $(2 / 8,45)$ & 6,64 & 35 & $\begin{array}{c}0,127-0,418 \\
(=)\end{array}$ & $\begin{array}{c}0,321-0,648 \\
(=)\end{array}$ & $\begin{array}{c}0,101-0,381 \\
(=)\end{array}$ \\
\hline $\begin{array}{l}\text { Lycalopex } \\
\text { griseus }\end{array}$ & $(44 / 21,03)$ & $(22 / 37,34)$ & $(11 / 18,61)$ & 34,49 & 77 & $\begin{array}{c}0,176-0,370 \\
(=)\end{array}$ & $\begin{array}{c}0,376-0,593 \\
(+)\end{array}$ & $\begin{array}{c}0,148-0,334 \\
(=)\end{array}$ \\
\hline $\begin{array}{l}\text { Galictis } \\
\text { cuja }\end{array}$ & $(17 / 6,55)$ & $(5 / 11,64)$ & $(2 / 5,80)$ & 22,93 & 24 & $\begin{array}{c}0,098-0,448 \\
(+)\end{array}$ & $\begin{array}{c}0,286-0,683 \\
(=)\end{array}$ & $\begin{array}{c}0,072-0,410 \\
(=)\end{array}$ \\
\hline
\end{tabular}

\section{DISCUSION}

La detección de fecas como método para estimación de presencia de carnívoros es una forma económica y factible de utilizar con especies difíciles de estudiar. Entre sus desventajas está la dificultad en la identificación de algunas especies (e.g., Lycalopex spp.) y algunos sesgos como la superposición en áreas de defecación. Más en general, implícito en estos análisis está la asunción de que las áreas de defecación coinciden con las áreas de alimentación, aun cuando el hallazgo de letrinas indicaría lo contrario (ver abajo).

\section{USO DE HÁBITAT}

La alta frecuencia de fecas de $P$. concolor en plantaciones forestales de especies exóticas se puede explicar por la distribución fragmentada que presentan las mismas, con numerosos espacios abiertos del tipo matorral/pradera, los cuales podrían ser mayormente utilizados por los pumas. Son pocos y contradictorios los estudios acerca del efecto de la fragmentación de bosque sobre la selección de hábitat de pumas (Nowell \& Jackson 1996; Courtin et al. 1980). Es necesario considerar que los rangos espaciales de actividad son particularmente grandes en los pumas, por lo que tendrían una percepción de grano fino de la heterogeneidad espacial, de tal modo que la fragmentación del paisaje no se manifestaría a la escala espacial de este estudio. Por ejemplo Dickson \& Beier (2002) estimaron, en el sur de
California (EE.UU.), un ámbito de hogar promedio de $93 \mathrm{~km}^{2}$ para hembras y de $363 \mathrm{~km}^{2}$ para machos. Pese a eso puede considerarse que también atributos específicos del paisaje (e.g., oferta de presas) pueden contribuir a la selección de hábitat; así el consumo de presa de los pumas en el área de estudio proviene tanto del bosque nativo ( $41,2 \%$ de $P$ Pudu puda), como del agroecosistema forestal (25,5\% lagomorfos) (véase Zúñiga et al. 2005).

El uso espacial de L. griseus sobre las plantaciones forestales, se puede explicar porque este agroecosistema contiene matorrales con abundante oferta de presas de pequeños mamíferos (e.g., roedores y lagomorfos), situación ya documentada para la costa de Concepción (Muñoz-Pedreros et al. 1990, Muñoz-Pedreros 1992). Así, en el área de estudio L. griseus consume, principalmente, Abrothrix longipilis, Oligoryzomys longicaudatus y Oryctolagus cuniculus (Zuñiga et al. 2005, 2008). Por otra parte, la presencia en el bosque nativo de fecas en forma de agregados (letrina), supondría una ocupación de este ambiente como zona nuclear, considerando su carácter territorial (Johnson \& Franklin 1994). Así, la flexible preferencia de hábitat de L. griseus es concordante con lo documentado por Acosta-Jamett \& Simonetti (2004) y González del Solar \& Rau (2004).

Los registros de fecas de L. guigna en el bosque nativo no son significativos, pero los registros de sus fecas en la plantación forestal son muy cercanos al bosque nativo $(10 \mathrm{~m})$, situación ya evidenciada en 
Gayana 73(2), 2009

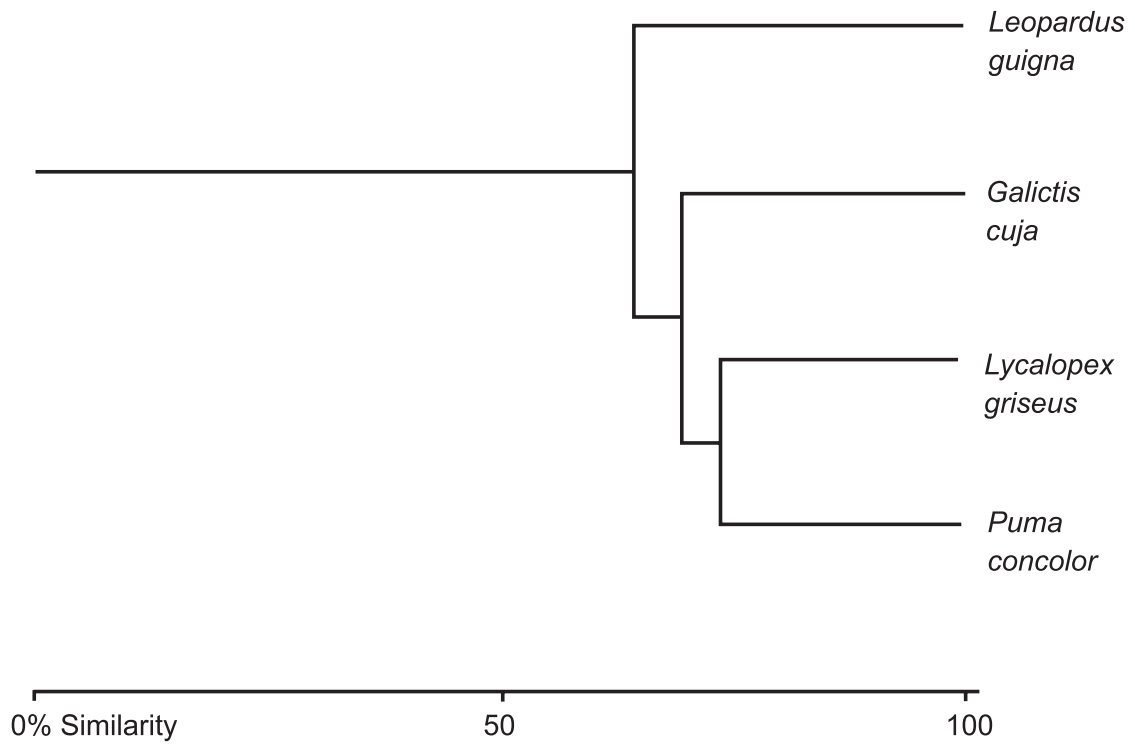

Figura 4. Similitud en el uso del hábitat para cuatro depredadores basados en los registros de frecuencias de fecas en tres hábitats del predio Rucamanque, Región de la Araucanía. 2005-2006.

FiguRE 4. Similarity of habitat use of four predators based on the records of frequencies of feces in three habitats at predio Rucamanque, Region de la Araucania. 2005-2006.

ambientes similares (Moraga 2005). En términos generales, el uso de L. guigna por el hábitat de bosque y sus proximidades, permiten asignarle una tendencia especialista (véase también Acosta-Jamett \& Simonetti 2004; Dunstone et al 2004), fuertemente vinculadas al dosel arbóreo (Konecny 1989), donde depreda sobre especies de hábitos arborícolas (e.g., aves, marsupiales, roedores).

Galictis cuja presenta una marcada preferencia por plantaciones forestales fragmentadas, donde los lagomorfos, uno de sus principales ítemes dietarios en el área de estudio (Zúñiga et al. 2005), son abundantes. Yensen \& Tarifa (2003) documentan, para esta especie, un uso generalista de ambientes; sin embargo, existen registros en mustélidos en los cuales éstos cambiarían su patrón de respuesta en función de los tamaños de los fragmentos en que ellos se presenten (Virgós 2002), lo que afectaría su manera de seleccionar hábitat. Se hace necesario, entonces, evaluar esta situación en G. cuja para poder determinar en forma más precisa los condicionantes del uso del espacio para esta especie.

Debe tenerse en cuenta que las plantaciones de pino insigne son hábitats ocupados por los carnívoros descritos cuando estos arreglos silvícolas no han cerrado su dosel, por lo que permiten la existencia de un sotobosque (matorral) que ofrece alimento y refugio tanto para los depredadores como para las presas (véase para Chile centro sur Muñoz-Pedreros \& Murúa 1989; Muñoz-Pedreros et al. 1990; MuñozPedreros 1992). Esto explicaría la mayor frecuencia de registros de P. concolor, G. cuja y L. griseus en la plantación de pino insigne. Situación que cambiaría drásticamente cuando el dosel se cierre y disminuya o desaparezca el sotobosque que contiene, pudiendo colapsar la ocurrencia de presas. De esta forma la plantación forestal bajo estudio es ecológicamente un sotobosque (véase Muñoz-Pedreros \& Murúa 1989) y esto explica su selección por la mayoría de las especies (salvo L. guigna), permitiendo su sobreposición espacial.

SOBREPOSICIÓN ESPACIAL

La co-ocurrencia de especies en un ambiente determinado está condicionada, principalmente, por sus tamaños y hábitos de vida (Dayan \& Simberloff 1998), existiendo una correspondencia entre el 
Uso de hábitat por carnívoros en el sur de Chile: ZúÑIGA, A. ET AL.
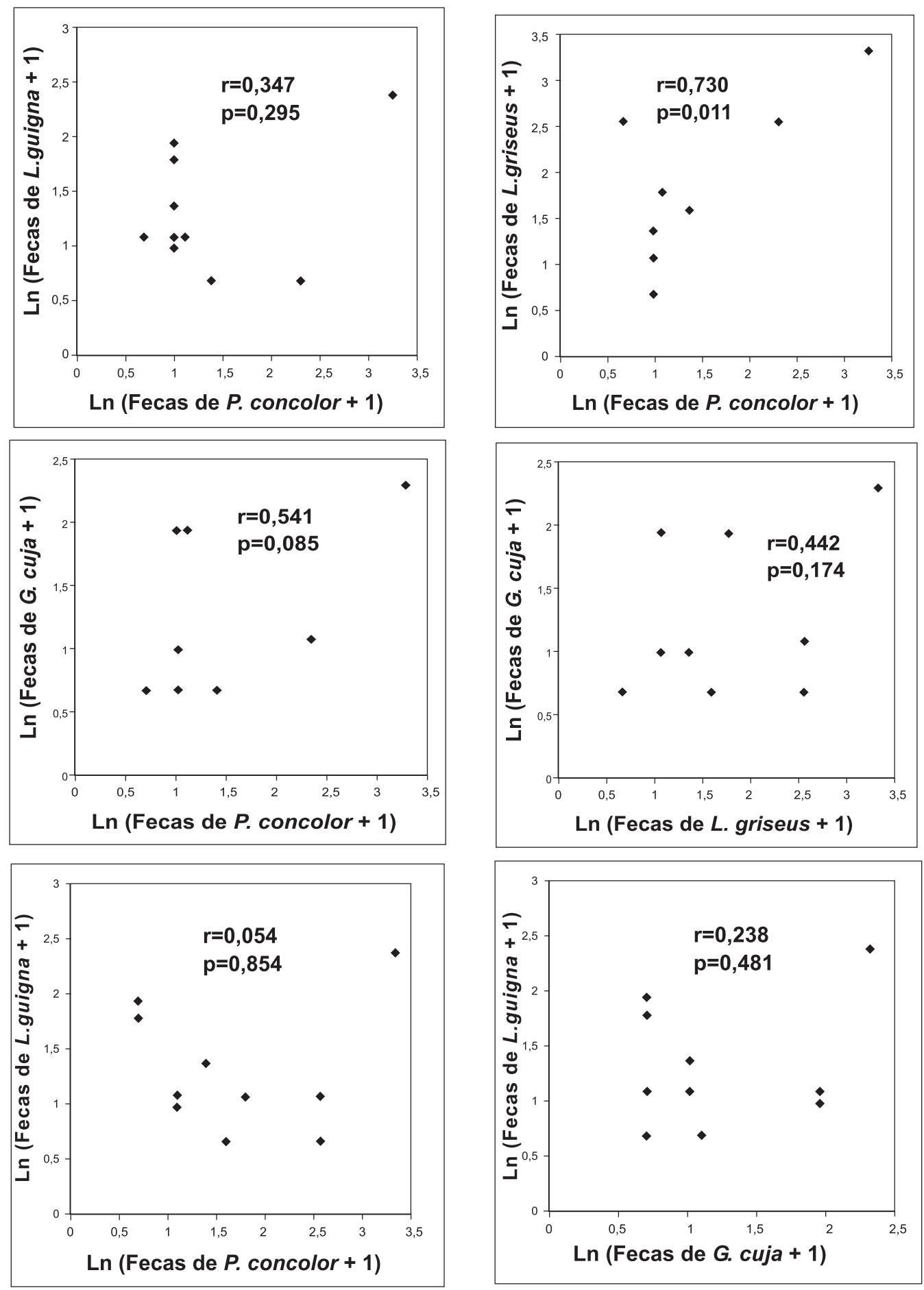

FiguRa 5. Sobreposición espacial, empleando el coeficiente de correlación de Pearson (r), considerando la frecuencia de fecas de cuatro carnívoros en 12 transectos en el predio Rucamanque, Región de la Araucanía. 2005-2006.

FIgURE 5. Spatial overlap, using the Pearson's correlation coefficient (r), among the frequencies of feces of four carnivores in 12 transects at predio Rucamanque, Region de la Araucania. 2005-2006. 
Gayana 73(2), 2009

tamaño corporal del depredador y el de la presa (Gittleman 1985). Así, en el área de estudio existe co-ocurrencia de especies de diferentes tamaños. Los resultados muestran una débil tendencia a la segregación, sin embargo esto debe considerarse con reserva ya que podría existir una sub-representación de las frecuencias (de fecas) en relación al área de estudio. Neale \& Sacks (2001) sugieren que esta sub-representación sería un sesgo metodológico cuando se emplean transectos definidos. Pese a esto, la baja sobreposición de $P$. concolor con los otros carnívoros puede explicarse por su tamaño mayor (y consecuentemente mayores presas), aun cuando podría ocurrir algún grado de competencia con L. griseus en las plantaciones forestales fragmentadas. Existe una mínima sobreposición entre $P$. concolor y L. guigna ya que este último prefiere los hábitats cerrados del bosque nativo, a diferencia de $P$. concolor que prefiere los hábitats abiertos (Novell \& Jackson 1996). Lycalopex griseus y $G$. cuja tienen diferentes tamaños y su sobreposición espacial no es significativa $(r=0,44)$, a la vez que presentan sobreposición trófica en el área de estudio (Zúñiga et al. 2005, 2008) en la que ambos consumen lagomorfos, situación análoga a la observada en Chile Central entre G. cuja y $L$. culpaeus (Ebensperger et al. 1991).

El co-uso espacial existente entre las especies en Rucamanque sugeriría que para mantener la estabilidad del ensamble, las especies que lo componen deben segregarse temporalmente (Koehler \& Hornocker 1991). Esto se ha argumentado para explicar la estabilidad entre depredadores mediterráneos con manifiesta sobreposición espacial (Fedriani et al. 1999). A pesar de que no existe evidencia en el área de estudio, la sobreposición de nichos podría derivar en acciones agonísticas excluyentes, que podrían determinar conductas de depredación intra-gremio (Palomares \& Caro 1999), lo que es necesario evaluar en el tiempo para determinar en forma más precisa los patrones de evolución ecológica que implica las sobreposiciones de nichos mencionadas.

\section{AGRADECIMIENTOS}

Los autores agradecen los comentarios al manuscrito de José Yáñez y Jaime Rau. También al Laboratorio de Ecología Aplicada y Biodiversidad de la Escuela de Ciencias Ambientales de la Universidad Católica de Temuco y a los revisores anónimos que mejoraron notablemente el manuscrito.

\section{BIBLIOGRAFIA}

Acosta-Jamett, G. \& Simonetti, J. 2004. Habitat use by Oncifelis guigna and Pseudalopex culpaeus in a fragmented forest landscape in central Chile. Biodiversity and Conservation 13 (6):11351151.

Byers, C. R., Steinhorst, R. K. \& Krausman, P. R. 1984. Clarification of a technique for analysis of utilization-availability data. Journal of Wildlife Management 48 (3):1050-1053.

Carroll, C., Noss, R. \& Paquet, P. 2001. Carnivores as focal species for conservation planning in the Rocky Mountain Region. Ecological Applications 11 (4):961-980.

Caughley, G. 1994. Directions in conservation biology. Journal of Animal Ecology 63 (2): 215-244.

Chame, M. 2003. Terrestrial mammal feces: a morphometric summary and description. Memoriás do Instituto Oswaldo Cruz 98 (1):71-94.

Courtin, S., Pacheco, L. \& Eldridge, W. 1980. Observaciones de alimentación, movimientos y preferencias de hábitat del puma en el islote Rupanco. Medio Ambiente 4:50-55.

Crooks, K.R. 2002. Relative sensitivities of mammalian carnivores to habitat fragmentation. Conservation Biology 16 (2):488-502.

Dayan, T. \& Simberloff, D. 1998. Size patterns among competitors: ecological character displacement and character release in mammals, with special reference to island populations. Mammal Review 28 (3):99-124.

Delibes, M., Traviani, A., Zapata, S. \& Palomares, F. 2003. Alien mammals and the trophic position of the lesser grison (Galictis cuja) in Argentinean Patagonia. Canadian Journal of Zoology 81 (1):157-162.

Dickson, B. \& P. Beier. 2002. Home-Range and habitat selection by adult cougars in Southern California. Journal of Wildlife Management 66 (4):12351245.

Dunstone, N., Freer, R., Acosta-Jamett, G., Durbin, I., Wyllie, J., Mazzolli, M. \& Scott, D. 2002. Uso del hábitat, actividad y dieta de la güiña (Oncifelis guigna) en el Parque Nacional Laguna San Rafael, XI Región, Chile. Boletín Museo Nacional de Historia Natural (Chile) 51:147-158.

Ebensperger L, Mella, J. \& Simonetti, J. 1991. Trophicniche relationships among Galictis cuja, Dusicyon culpaeus and Tyto alba in Central Chile. Journal of Mammalogy 72 (4): 820-823.

Echeverría, C., Coomes, D., Rey-Benayas, J.M., Lara, A. \& Newton, A. 2006. Rapid deforestation and fragmentation of Chilean temperate forests. Biological conservation 130 (4):481-494.

Echeverrí́a, C., Coomes, D., Hall, M. \& Newton, A. 
Uso de hábitat por carnívoros en el sur de Chile: ZúÑIGA, A. ET AL.

2008. Spatially explicit models to analyze forest loss and fragmentation between 1976 and 2020 in southern Chile. Ecological Modelling 212 (3-4):439-449.

Fedriani, J., Palomares, F. \& Delibes, M. 1999. Niche relations among three sympatric mediterranean carnivores. Oecologia 121 (1): 138-148.

Gehring, T. \& Swinhart, R. 2003. Body size, niche breadth and ecologically scaled responses to habitat fragmentation: mammalian predators in an agricultural landscape. Biological Conservation 109 (2): 283-295.

Gittleman, J. 1985. Carnivore body size: ecological and taxonomical correlates. Oecologia 67 (4):540554.

GonzÁlez del Solar, R. \& Rau, J. 2004. Chilla, In: Canids: Foxes, Wolves, Jackals and Dog: Status Survey and Conservation Action Plan (Eds. Sillero-Zubiri, C., Hoffmann, M. \& D.W. MacDonald), pp. 53-56. IUCN Publication Service Unit, Cambridge.

Guerrero, C., Espinoza, L., Niemeyer, H. \& Simonetti, J. 2006. Using fecal profiles to assess habitat use by threatened carnivores in the Maulino forest of central Chile. Revista Chilena de Historia Natural 79 (1): 85-89.

Holm, S. 1979. A simple sequentially rejective multiple test procedure. Scandinavian Journal of Statistics 6 (1): 65-70.

JAKSIC, F. \& MedeL, R. 1987. El acuchillamiento de datos como método de obtención de intervalos de confianza y de prueba de hipótesis para índices ecológicos. Medio Ambiente 8 (2): 95-103.

Johnson, W. \& Franklin, W. 1994. Spatial resource partitioning by sympatric grey fox (Dusicyon griseus) and culpeo fox (Dusicyon culpaeus) in southern Chile. Canadian Journal of Zoology 72 (10):1788-1793.

Kleiman, D. \& Eisenberg, J. 1973. Comparison of canid and felid social systems from an evolutionary perspective. Animal Behaviour 21 (4):637-659.

Koehler, G. \& Hornocker, M. 1991. Seasonal resource use among mountain lions, bobcats, and coyotes. Journal of Mammalogy 72 (2): 391-396.

KoneCNy, M. 1989. Movement patterns and food habits in four sympatric carnivore species in Belize, Central America. Advances in Neotropical Mammalogy. Brill, Leiden. 243-264 pp.

LinDENMAYER, D. \& FisCHER, J. 2006. Habitat fragmentation and landscape change: an ecological and conservation synthesis. University of Chicago Press, Chicago. 328 pp.

Magofke, J. 1985. Rucamanque: un relicto de bosque en Temuco, Chile. Revista Frontera (Temuco) 4:65-72.

Mc Aleece, N. 1997. Biodiversity professional beta 1 . Version 2. The Natural History Museum \& The Scottish Association for Marine Science. http_// www.nhm.ac.uk.uk/zoology/bdpro.

Moraga, C. 2005. Prospección de güiña, Oncifelis guigna (felidae) y zorro culpeo, Pseudalopex culpaeus
(Canidae) en plantaciones forestales de pino (Pinus radiata D. Don) de la Cordillera de la Costa de la provincia de Ñuble, Región del Biobío. Tesis de Licenciatura. Facultad de Medicina Veterinaria, Universidad de Concepción.

MuÑoz, M, NúÑEz, H. \& YÁÑEZ J., EdS. 1996. Libro rojo de los sitios prioritarios para la conservación de la diversidad biológica en Chile. Ministerio de Agricultura, Corporación Nacional Forestal. Santiago. 203 pp.

Muñoz-Pedreros, A. 1992. Ecología del ensamble de micromamíferos en un agroecosistema forestal de Chile central. Una comparación latitudinal. Revista Chilena Historia Natural 65 (4):417428.

Muñoz-Pedreros, A. \& Murúa, R. 1989. Efectos de la reforestación con Pinus radiata sobre la diversidad y abundancia de los micromamíferos en la zona costera central de Chile. Turrialba (Costa Rica) 59: 143-150.

Muñoz-Pedreros, A., Murúa, R. \& González, L. 1990. Nicho ecológico de micromamíferos presentes en un agroecosistema forestal de Chile central. Revista Chilena de Historia Natural 63 (3):267277.

Murray, D., Boutin, L.S., O’Donnoghue, M. \& Nams, V.O. 1995. Hunting behaviour of sympatric felids and canids in relation to vegetative cover. Animal Behaviour 50 (5):1203-1210.

Neale, J. \& Sacks, B. 2001. Food habits and space use of gray foxes in relation to sympatric coyotes and bobcats. Canadian Journal of Zoology 79 (10): 1794-1800.

Nowell, K. \& JACKSON, P. 1996. Wildcats: status survey and conservation action plan. Gland, Switzerland, IUCN. 382 pp.

Odum, E. 1950. Bird populations of the Highlands (North America) Plateau in relation to plant succession and avian invasion. Ecology 31 (4): 587-605.

Palomares, F. \& Caro, T. 1999. Interespecific killing among mammalian carnivores. American Naturalist 153 (5): 492-508.

Quintana, V., Yáñez, J., Valdebenito M. \& Iriarte A. 2009. Carnívoros. En: Mamíferos de Chile (Eds. Muñoz-Pedreros A. \& J. Yáñez), pp. 193-230. CEA Ediciones, Valdivia.

RAMíREZ, C. 1982. Pasado, presente y futuro: La vegetación nativa del sur de Chile. Creces 3 (6-7): 40-45.

Ramírez C, Hauenstein, E., San Martín, J, \& Contreras. D. 1989a. Study of the flora of Rucamanque, Cautín Province, Chile. Annals of the Missouri Botanical Garden 76: 444-453.

Ramírez, C., Hauenstein, E., San Martín, J. \& Contreras, D. 1989b. Estudio fitosociológico de la vegetación de Rucamanque (Cautín, Chile). Stvdia Botánica 8: 91-115.

Sadlier L, Webbon, C., Baker, P. \& Harris, S. 2004. Methods of monitoring red foxes Vulpes vulpes and badgers Meles meles: are field signs the answer? Mammal Review 34(1-2):1-25.

SAn Martín, J. \& Donoso C. 1996. Estructura florística 
Gayana 73(2), 2009

e impacto antrópico en el bosque Maulino de Chile. En: Ecología de los bosques nativos de Chile (Eds. Armesto, J.J., Villagrán, C. \& C.M.K. Arroyo), pp.153-168. Editorial Universitaria, Santiago, Chile.

Sánchez-Lalinde, C. \& Pérez-Torres, J. 2008. Uso de hábitat de carnívoros simpátricos en una zona de bosque seco tropical de Colombia. Mastozoología Neotropical 15 (1): 167-174.

Skole, D. \& Tucker, C. 1993. Tropical deforestation and habitat fragmentation in the Amazon: satellite data from 1978 to 1988. Science 260: 1905-1910.

SoKal, R.R. \& Rohlf F.J. 1995. Biometry. W.H. Freeman and Company, New York. 887 pp.

VIRGÓs, E. 2002. Are habitat generalists affected by forest fragmentation? A test with Eurasian badgers (Meles meles) in coarse-grained fragmented landscapes of central Spain. Journal of Zoology (London) 258 (3): 313-318.

Virgós, E., Tillería, J.L. \& Santos, T. 2002. A comparison on the response to forest fragmentation by medium-sized Iberian carnivores in central Spain. Biodiversity and Conservation 11 (6): 1063-1079.

YÁÑez, J. \& Muñoz-Pedreros, A. 2009. Mamíferos vivientes de Chile. En: Mamíferos de Chile (Eds. Muñoz-Pedreros A. \& J. Yáñez), pp. 47-50. CEA Ediciones, Valdivia.

Yensen, E. \& Tarifa, T. 2003. Galictis cuja. Mammalian Species 728:1-8.

ZAR, J. 1984. Biostatistical analisis. 2nd. ed. Prentixe Hall Inc., Englewood Cliffs, N. J. 718 pp.

ZúÑIga, A., Quintana, V. \& Fierro, A. 2005. Relaciones tróficas entre depredadores en un ambiente fragmentado del sur de Chile. Gestión Ambiental 11:31-42.

ZúÑIga, A., Muñoz-Pedreros, A. \& Fierro, A. 2008. Dieta de Lycalopex griseus (Mammalia: Canidae) en la depresión intermedia del sur de Chile. Gayana 72 (1): 167-170.

Recibido: 16.12 .08

Aceptado: 04.10.09 